\title{
Knowledge and practice of university students in Lebanon regarding contraception
}

B. Barbour ${ }^{1}$ and P. Salameh ${ }^{2}$

$$
\begin{aligned}
& \text { معارف طلبة الجامعات في لبنان وممارساتهم حول منع الحمل }
\end{aligned}
$$

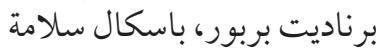

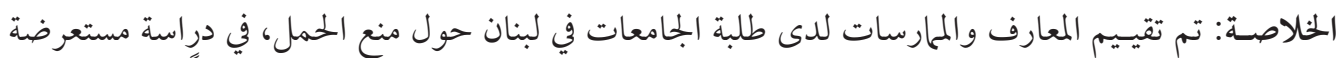

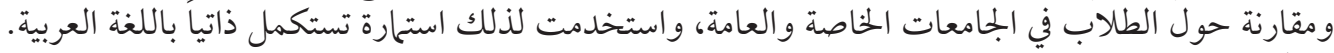

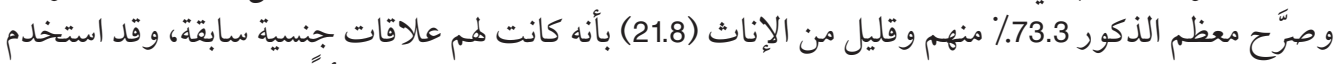

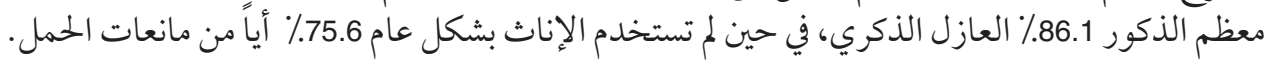

ABSTRACT We evaluated knowledge and practice of Lebanese university students regarding contraception in a cross-sectional, comparative study on students in public and private universities using an Arabic language self-administered questionnaire. We found low levels of knowledge of contraception. The majority of males $(73.3 \%)$ and a few females $(21.8 \%)$ declared previous sexual relations: the majority of males had used a condom (86.1\%), but females had generally not used contraceptives $(75.6 \%)$.

\section{Connaissances et pratiques en matière de contraception des étudiants libanais}

RÉSUMÉ Nous avons évalué les connaissances et les pratiques en matière de contraception des étudiants libanais dans le cadre d'une étude transversale comparative réalisée auprès d'étudiants d'universités publiques et privées sur la base d'un questionnaire en langue arabe à remplir soi-même. Le niveau des connaissances était faible. La majorité des garçons $(73,3 \%)$ et peu de filles $(21,8 \%)$ ont déclaré avoir déjà eu des rapports sexuels : la majorité des garçons avaient utilisé un préservatif $(86,1 \%)$, mais les filles n'avaient généralement pas utilisé de contraceptifs $(75,6 \%)$.

\footnotetext{
${ }^{1}$ Faculty of Public Health Section II; ${ }^{2}$ Faculty of Pharmacy, Lebanese University, Beirut, Lebanon (Corrrespondence to P. Salameh: pascalesalameh1@yahoo.com; psalameh@ul.edu.lb).

Received: 28/05/06; accepted: 13/09/06
} 


\section{Introduction}

Public health policies and programmes are nowadays focusing on the sexual and reproductive health needs of adolescents, particularly in the developing world [1]. Adolescents are not always rational when making sexual choices: being deeply involved in their own bodily perceptions, strong emotions and feelings of ambivalence may override the perception of risk [2], despite the fact that attitude towards behaviour generally reflects an individual's beliefs and consequences associated with engaging in the behaviour [3]. Thus, sexual habits, contraceptive use and sexually transmitted disease (STD) rank among the most important health issues for adolescents and young adults. In addition, early sexual activity is associated with risky behaviours such as smoking, alcohol use, multiple sexual partners and unintended pregnancy [4]. Emerging research suggests that teenagers make decisions about contraceptive use in the context of individual sexual relationships [5]. Different beliefs in males and females may also affect contraceptive use and need to be explored to develop sex education and services for this age group [6].

Media and friends, not health professionals, have been reported as the primary sources of information for young women and men of all ages [7]. Hence, inadequate information is expected in youngsters. For example, in a study on Ghanaian youth, nearly all respondents (99\%) knew of condoms but less than half (48\%) could identify any of 4 elements of correct use; females and sexually inexperienced youth were the least informed [8]. In a representative sample of individuals aged 16-45 years in Greece, only a small percentage of the respondents were able to answer correctly $50 \%$ or more of the questions on knowledge of basic contraceptive issues $(30.6 \%$ of women and
$14.7 \%$ of men), although the majority of respondents considered themselves at least adequately informed [7].

Lebanon is a developing country with conservative norms, particularly for girls, and little information exists regarding contraception. Nevertheless, there are actual changes in social, cultural and moral norms, with large discrepancies between religions. These changes seem to exert a considerable effect on the country's young adult population. It is generally observed that young people tend to engage in sexual activity at younger ages than before; the use of contraception in these settings is largely unknown. This study, therefore, aimed to evaluate knowledge, attitudes and practices of Lebanese university students regarding contraception, in a comparative analysis between males and females.

\section{Methods}

This was a cross-sectional comparative study. The sampling frame was a list of departments of all public and private universities in Lebanon, from which a random sample of 15 was drawn up. In the public university (Lebanese University) we sampled the faculties of arts, law and political sciences, public health, engineering, information and documentation, social sciences, literature and humanities, and sciences. The private universities selected were Kaslik Holy Spirit University, Saint Joseph University (Uvelain campus), American University of Science \& Technology (Achrafieh campus), Notre Dame University (Loueizeh campus), Beirut Arab University, American University of Beirut and Balamand University (Tripoli campus).

Campus administrators were contacted and permission was given to enquirers to distribute questionnaires in all but the Amer- 
ican University of Beirut campus, where permission to participate in the study was refused. A convenience sample of available students was chosen to participate to the study: a lay enquirer was sent to spend the day on campus and distribute a minimum of 100 questionnaires per campus. Students were approached during recess hours. Exclusion criteria were being married or of non-Lebanese nationality.

A self-administered standardized questionnaire was used, to be completed in the local Arabic language on campus. Closed and open-ended questions were asked. The questionnaire was based on current knowledge of available contraceptives and STDs; information collected was similar to that gathered by other authors [7-9]. It was divided into 5 parts: social and demographic characteristics; knowledge regarding commonly used contraceptives; knowledge regarding the menstrual cycle and natural fertility regulation; practices regarding contraceptive use and failure; and knowledge about STDs. The questionnaire was pilottested on 10 young individuals aged 18-22 years for correcting or clarifying questions when necessary.

The study was carried out between April 2005 and June 2005. Students gave oral consent to participate to the study, after explaining that it was a "study done by university researchers that had extreme importance for their health" and ensuring anonymity (no names were required). To ensure maximum objectivity in students' answers, enquirers were instructed not to give any additional clarification for questions which were not understood.

Questionnaires were coded and data entered on SPSS, version 12.0, by independent lay persons. Data entry was then controlled, and data analysed using the same software. $P$-value $<0.05$ was considered significant. Missing values, which accounted for $<20 \%$ of answers, were not replaced, and variables were analysed as available. The chi-squared test was used for comparison between categorical variables. Analysis of variance was used to compare means of continuous variables.

\section{Results}

We distributed 2000 questionnaires and $1410(70.5 \%)$ were returned. There were some differences in the social and demographic characteristics of the male and female respondents: there were more female respondents from public universities $(P$ $<0.0001)$, more Christians $(P<0.002)$ and more people living in Mount Lebanon $(P$ $<0.02$ ). Males were slightly, but statistically significantly, older than females $(P$ $<0.0001)$. No statistically significant differences were noted for study year or region of origin $(P>0.05)$ (Table 1).

\section{Knowledge regarding contraceptives}

Books (57.5\%), friends (56.2\%) and school $(52.0 \%)$ were the most cited sources of information on sex (Table 2). No respondent cited any health professional.

All males knew about the condom, but $2.8 \%$ of females had never heard of it $(P$ $<0.0001)$. Males knew more about the conditions of use, contraindications and side-effects of condoms $(P<0.0001)$. Only half the male respondents regularly verified the expiry date before using a condom and knew when to put it on, while less than onethird knew when to remove it (Table 3).

Three-quarters of males had heard about the intrauterine device (IUD) in comparison with $88.6 \%$ of females $(P<0.0001)$. Onethird of respondents thought that an IUD was placed in the vagina, $10.7 \%$ thought it could be used by any woman, and $15.0 \%$ did not know how it is used. Significantly

المجلة الصحية لشرق المتوسط، منظمة الصحة العالمية، المجلد الخامس عشر، العدد Y، 9.ب. 
Table 1 Social and demographic characteristics of participants

\begin{tabular}{lccccccc}
\hline Characteristic & \multicolumn{2}{c}{ Males $(\boldsymbol{n}=\mathbf{5 0 5})$} & \multicolumn{2}{c}{ Females $(\boldsymbol{n}=\mathbf{9 0 5})$} & \multicolumn{2}{c}{$\boldsymbol{P}$-value } & \multicolumn{2}{c}{ Total $(\boldsymbol{n}=\mathbf{1 4 1 0})$} \\
& No. & $\%$ & No. & $\%$ & & No. & $\%$ \\
\hline University & & & & & & & \\
$\quad$ Public & 290 & 57.4 & 707 & 78.1 & $<0.0001$ & 997 & 70.7 \\
$\quad$ Private & 215 & 42.6 & 198 & 21.9 & & 413 & 29.3 \\
Study year & & & & & & & \\
$\quad$ Undergraduate & 395 & 78.2 & 742 & 82.0 & 0.17 & 1137 & 80.6 \\
$\quad$ Graduate & 88 & 17.4 & 131 & 14.5 & & 219 & 15.5 \\
$\quad$ Postgraduate & 6 & 1.2 & 18 & 2.0 & & 24 & 1.7 \\
Region of origin & & & & & & & \\
$\quad$ Beirut & 68 & 13.5 & 92 & 10.2 & 0.09 & 160 & 11.3 \\
$\quad$ Mount Lebanon & 204 & 40.4 & 407 & 45.0 & & 611 & 43.3 \\
$\quad$ Other & 233 & 46.1 & 406 & 44.9 & & 639 & 45.3 \\
Residence & & & & & & & \\
$\quad$ Beirut & 85 & 16.8 & 108 & 11.9 & 0.02 & 193 & 13.7 \\
$\quad$ Mount Lebanon & 351 & 69.5 & 689 & 76.1 & & 1040 & 73.8 \\
$\quad$ Other & 69 & 13.7 & 108 & 11.9 & & 177 & 12.6 \\
Religion & & & & & & & \\
$\quad$ Christian & 390 & 77.2 & 764 & 84.4 & 0.002 & 1154 & 81.8 \\
$\quad$ Muslim & 49 & 9.7 & 68 & 7.5 & & 117 & 8.3 \\
$\quad$ No answer & 66 & 13.1 & 73 & 8.1 & & 139 & 9.9 \\
Mean (SD) age (years) & $21.0(1.9)$ & $20.3(1.6)$ & $<0.0001$ & $20.6(1.8)$ \\
\hline SD
\end{tabular}

$S D=$ standard deviation.

more females than males knew that IUDs have side-effects and contraindications $(P$ $<0.0001$ ) (Table 3).

About half the respondents had ever heard about the cervical cap, significantly more females than males $(P<0.0001)$. The majority of these, however, did not know how it is used, and more than $80 \%$ did not

Table 2 Stated source of sexual education for university students in Lebanon

\begin{tabular}{lcc}
\hline $\begin{array}{l}\text { Source of } \\
\text { information }\end{array}$ & No. $(\boldsymbol{n}=\mathbf{1 4 1 0})$ & $\%$ \\
\hline Books & 811 & 57.5 \\
Friends & 792 & 56.2 \\
School & 733 & 52.0 \\
Mass media & 541 & 38.4 \\
Parents & 420 & 29.8 \\
Partners & 378 & 26.8 \\
University & 283 & 20.1 \\
\hline
\end{tabular}

know if it has side-effects or how long it should be left in place. Similar findings applied to the vaginal diaphragm (Table 3).

Almost all females $(97.9 \%)$ and males (88.1\%) declared knowing about oral contraception (Table 4). However, about half the responding males and a third of the females did not know how frequently pills are used $(P<0.003)$. Just over half the students stated they did not know what to do in the case of a forgotten dose. More females than males were aware of the existence of side-effects and contraindications for oral contraceptives $(P<0.0001)$ (Table 4$)$.

Two-thirds of respondents declared knowing about spermicidal products, but the majority of females did not know how they are used or the side-effects $(P<0.0001)$. Even males who declared that they knew how to use them had erroneous information (Table 4). 
Table 3 Knowledge of students regarding mechanical contraceptives

\begin{tabular}{|c|c|c|c|c|c|c|c|}
\hline \multirow{2}{*}{$\begin{array}{l}\text { Type of contraceptive \& } \\
\text { knowledge item }\end{array}$} & \multicolumn{2}{|c|}{ Males $(n=505)$} & \multicolumn{2}{|c|}{ Females $(n=905)$} & \multirow[t]{2}{*}{$P$-value } & \multicolumn{2}{|c|}{ Total $(n=1410)$} \\
\hline & No. & $\%$ & No. & $\%$ & & No. & $\%$ \\
\hline \multicolumn{8}{|l|}{ Male condom } \\
\hline Declare knowing about it & 505 & 100 & 880 & 97.2 & $<0.0001$ & 1385 & 98.2 \\
\hline Never used it & 46 & 9.1 & 481 & 54.7 & & 527 & 38.1 \\
\hline Is disposable & 248 & 49.1 & 418 & 47.5 & 0.94 & 666 & 48.1 \\
\hline Necessary to verify expiry date & 472 & 93.5 & 816 & 92.7 & 0.09 & 1288 & 93.0 \\
\hline \multicolumn{8}{|l|}{ Is used } \\
\hline On erect penis & 441 & 87.3 & 749 & 85.1 & 0.87 & 1190 & 85.9 \\
\hline At the beginning of erection & 269 & 53.3 & 260 & 29.5 & $<0.0001$ & 529 & 38.2 \\
\hline Just before ejaculation & 39 & 7.7 & 47 & 5.3 & 0.10 & 86 & 6.2 \\
\hline Do not know & 20 & 4.0 & 271 & 30.8 & $<0.0001$ & 291 & 21.0 \\
\hline \multicolumn{8}{|l|}{ Is removed } \\
\hline While penis still erect & 149 & 29.5 & 85 & 9.4 & $<0.0001$ & 234 & 16.9 \\
\hline On detumescence & 304 & 60.2 & 376 & 42.7 & $<0.0001$ & 680 & 49.1 \\
\hline Do not know when to remove & 44 & 8.7 & 373 & 41.2 & $<0.0001$ & 417 & 30.1 \\
\hline \multicolumn{8}{|l|}{ Verifies expiry date } \\
\hline Always & 284 & 56.2 & 237 & 26.9 & $<0.0001$ & 521 & 37.6 \\
\hline Sometimes & 126 & 25.0 & 78 & 8.9 & & 204 & 14.7 \\
\hline Never & 44 & 8.7 & 33 & 3.8 & & 77 & 5.6 \\
\hline \multicolumn{8}{|l|}{ Contraindications? } \\
\hline Yes & 106 & 21.0 & 165 & 18.8 & $<0.0001$ & 271 & 19.6 \\
\hline No & 214 & 42.4 & 200 & 22.7 & & 414 & 29.9 \\
\hline Do not know & 176 & 34.9 & 477 & 54.2 & & 653 & 47.1 \\
\hline \multicolumn{8}{|l|}{ Side-effects? } \\
\hline Yes & 82 & 16.2 & 121 & 13.8 & $<0.0001$ & 203 & 14.7 \\
\hline No & 238 & 47.1 & 251 & 28.5 & & 489 & 35.3 \\
\hline Do not know & 169 & 33.5 & 455 & 51.7 & & 624 & 45.1 \\
\hline \multicolumn{8}{|l|}{ Intrauterine device } \\
\hline Declares knowing about it & 366 & 72.5 & 802 & 88.6 & $<0.0001$ & 1168 & 82.8 \\
\hline Is placed in the uterus & 134 & 36.6 & 390 & 48.6 & $<0.0001$ & 524 & 44.9 \\
\hline Is in place for a long time & 100 & 27.3 & 330 & 41.1 & $<0.0001$ & 430 & 36.8 \\
\hline Is placed in the vagina & 132 & 36.1 & 247 & 30.8 & 0.08 & 379 & 32.4 \\
\hline Can be used by any woman & 43 & 11.7 & 82 & 10.2 & 0.42 & 125 & 10.7 \\
\hline Is used before every intercourse & 30 & 8.2 & 27 & 3.4 & $<0.0001$ & 57 & 4.9 \\
\hline Is placed during menstruation & 20 & 5.5 & 30 & 3.7 & 0.18 & 50 & 4.3 \\
\hline Does not know how it is used & 58 & 15.8 & 117 & 14.6 & 0.57 & 175 & 15.0 \\
\hline \multicolumn{8}{|l|}{ Side-effects? } \\
\hline Yes & 118 & 32.2 & 410 & 51.1 & $<0.0001$ & 528 & 45.2 \\
\hline No & 64 & 17.5 & 89 & 11.1 & & 153 & 13.1 \\
\hline Do not know & 173 & $\underline{47.3}$ & 291 & 36.3 & -- & 464 & 39.7 \\
\hline
\end{tabular}


Table 3 Knowledge of students regarding mechanical contraceptives (concluded)

\begin{tabular}{|c|c|c|c|c|c|c|c|}
\hline \multirow{2}{*}{$\begin{array}{l}\text { Type of contraceptive \& } \\
\text { knowledge item }\end{array}$} & \multicolumn{2}{|c|}{ Males $(n=505)$} & \multicolumn{2}{|c|}{ Females $(n=905)$} & \multirow[t]{2}{*}{$P$-value } & \multicolumn{2}{|c|}{ Total $(n=1410)$} \\
\hline & No. & $\%$ & No. & $\%$ & & No. & $\%$ \\
\hline \multicolumn{8}{|l|}{ Contraindications? } \\
\hline Yes & 58 & 15.8 & 250 & 31.2 & \multirow[t]{3}{*}{$<0.0001$} & 308 & 26.4 \\
\hline No & 55 & 15.0 & 460 & 57.4 & & 515 & 44.1 \\
\hline Do not know & 231 & 63.1 & 58 & 7.2 & & 289 & 24.7 \\
\hline \multicolumn{8}{|l|}{ Cervical cap } \\
\hline Declares knowing about it & 233 & 46.1 & 544 & 60.1 & $<0.0001$ & 777 & 55.1 \\
\hline Is disposable & 40 & 17.2 & 82 & 15.1 & 0.48 & 122 & 15.7 \\
\hline Changed after every intercourse & 31 & 13.3 & 81 & 14.9 & 0.55 & 112 & 14.4 \\
\hline Is used with a spermicide & 30 & 12.9 & 59 & 10.8 & 0.43 & 89 & 11.5 \\
\hline Is washable & 40 & 17.2 & 44 & 8.1 & $<0.0001$ & 84 & 10.8 \\
\hline Does not know about use & 126 & 54.1 & 361 & 66.4 & 0.001 & 487 & 62.7 \\
\hline \multicolumn{8}{|l|}{ Side-effects? } \\
\hline Yes & 25 & 10.7 & 53 & 9.7 & \multirow[t]{3}{*}{0.01} & 78 & 10.0 \\
\hline No & 31 & 13.3 & 38 & 7.0 & & 69 & 8.9 \\
\hline Do not know & 161 & 69.1 & 432 & 79.4 & & 593 & 76.3 \\
\hline \multicolumn{8}{|l|}{ Can be left in place for } \\
\hline 2 days & 44 & 18.9 & 59 & 10.8 & 0.003 & 103 & 13.3 \\
\hline 1 week & 21 & 9.0 & 17 & 3.1 & 0.001 & 38 & 4.9 \\
\hline 1 month & 13 & 5.6 & 13 & 2.4 & 0.025 & 26 & 3.3 \\
\hline Do not know & 146 & 62.7 & 421 & 77.4 & $<0.0001$ & 567 & 73.0 \\
\hline \multicolumn{8}{|l|}{ Vaginal diaphragm } \\
\hline Declares knowing about it & 262 & 51.9 & 583 & 64.4 & $<0.0001$ & 845 & 59.9 \\
\hline Is disposable & 55 & 21.0 & 144 & 24.7 & 0.24 & 199 & 23.6 \\
\hline Changed after every intercourse & 51 & 19.5 & 116 & 19.9 & 0.88 & 167 & 19.8 \\
\hline Is used with a spermicide & 53 & 20.2 & 108 & 18.5 & 0.57 & 161 & 19.1 \\
\hline Is washable & 48 & 18.3 & 46 & 7.9 & $<0.0001$ & 94 & 11.1 \\
\hline Does not know about its use & 103 & 39.3 & 323 & 55.4 & $<0.0001$ & 426 & 50.4 \\
\hline \multicolumn{8}{|l|}{ Side-effects? } \\
\hline Yes & 30 & 11.5 & 80 & 13.7 & $<0.0001$ & 110 & 13.0 \\
\hline No & 47 & 17.9 & 45 & 7.7 & & 92 & 10.9 \\
\hline Do not know & 166 & 63.4 & 437 & 75.0 & & 603 & 71.4 \\
\hline
\end{tabular}

Details about contraceptive type were filled in only by those who declared knowing about the contraceptive in question; percentages were calculated accordingly.

Some totals $<100 \%$ because of missing values.

\section{Knowledge about sexually transmitted diseases}

More females than males knew about STDs $(P<0.0001)$, but more females than males stated they did not know how to prevent them. About $80 \%$ thought that the use of condoms may prevent STDs, while others cited erroneous measures (Table 5).

\section{Knowledge regarding menstrual cycle and natural fertility regulation}

We found low levels of knowledge in both male and female respondents regarding the menstrual cycle and natural fertility regulation (Table 6). The majority, males in particular, did not know what the menstrual cycle is, when ovulation occurs or when ab- 


\begin{tabular}{|c|c|c|c|c|c|c|c|}
\hline \multirow{2}{*}{$\begin{array}{l}\text { Type of contraceptive \& } \\
\text { knowledge item }\end{array}$} & \multicolumn{2}{|c|}{ Males $(n=505)$} & \multicolumn{2}{|c|}{ Females $(n=905)$} & \multirow[t]{2}{*}{$P$-value } & \multicolumn{2}{|c|}{ Total $(n=1410)$} \\
\hline & No. & $\%$ & No. & $\%$ & & No. & $\%$ \\
\hline \multicolumn{8}{|l|}{ Oral contraceptive } \\
\hline Declares knowing about it & 445 & 88.1 & 886 & 97.9 & $<0.0001$ & 1331 & 94.4 \\
\hline Necessary to verify expiry date & 417 & 93.7 & 855 & 96.5 & 0.03 & 1272 & 95.6 \\
\hline \multicolumn{8}{|l|}{ Frequency of use } \\
\hline 1st-21st day of cycle & 88 & 19.8 & 255 & 28.8 & 0.003 & 343 & 25.8 \\
\hline Every day & 71 & 16.0 & 167 & 18.8 & 0.46 & 238 & 17.9 \\
\hline 5th-25th day of cycle & 52 & 11.7 & 159 & 17.9 & 0.01 & 211 & 15.9 \\
\hline At a fixed hour & 47 & 10.6 & 176 & 19.9 & $<0.0001$ & 223 & 16.8 \\
\hline Do not know about use & 181 & 40.7 & 311 & 35.1 & 0.003 & 492 & 37.0 \\
\hline \multicolumn{8}{|l|}{ In case of forgetting one tablet } \\
\hline Skip the forgotten tablet & 64 & 14.4 & 162 & 18.3 & 0.25 & 226 & 17.0 \\
\hline Take forgotten tablet soon & 38 & 8.5 & 115 & 13.0 & 0.06 & 153 & 11.5 \\
\hline Take both tablets together & 26 & 5.8 & 91 & 10.3 & 0.02 & 117 & 8.8 \\
\hline Do not know what to do & 267 & 60.0 & 489 & 55.2 & $<0.0001$ & 756 & 56.8 \\
\hline \multicolumn{8}{|l|}{ Are there several types of pill? } \\
\hline Yes & 139 & 31.2 & 453 & 51.1 & $<0.0001$ & 592 & 44.5 \\
\hline No & 25 & 5.6 & 39 & 4.4 & & 64 & 4.8 \\
\hline Do not know & 251 & 56.4 & 378 & 42.7 & & 629 & 47.3 \\
\hline Cannot be used at all ages & 173 & 38.9 & 372 & 42.0 & $<0.0001$ & 545 & 40.9 \\
\hline $\begin{array}{l}\text { Cannot be used by all women } \\
\text { Side-effects? }\end{array}$ & 212 & 47.6 & 549 & 62.0 & $<0.0001$ & 761 & 57.2 \\
\hline Yes & 181 & 40.7 & 537 & 60.6 & $<0.0001$ & 718 & 53.9 \\
\hline No & 30 & 6.7 & 43 & 4.9 & & 73 & 5.5 \\
\hline Do not know & 188 & 42.2 & 276 & 31.1 & & 464 & 34.9 \\
\hline \multicolumn{8}{|l|}{ Spermicide } \\
\hline $\begin{array}{l}\text { Declares knowing about it } \\
\text { How used }\end{array}$ & 319 & 63.2 & 598 & 66.1 & 0.42 & 917 & 65.0 \\
\hline $\begin{array}{l}\text { How used } \\
15 \text { min before intercourse }\end{array}$ & 91 & 28.5 & 88 & 14.7 & $<0.0001$ & 179 & 19.5 \\
\hline Just before intercourse & 53 & 16.6 & 91 & 15.2 & 0.57 & 144 & 15.7 \\
\hline $2 \mathrm{~h}$ before intercourse & 61 & 19.1 & 34 & 5.7 & $<0.0001$ & 95 & 10.4 \\
\hline After ejaculation & 10 & 3.1 & 7 & 1.2 & 0.04 & 17 & 1.9 \\
\hline In the vagina & 63 & 19.7 & 98 & 16.4 & 0.20 & 161 & 17.6 \\
\hline On external genitalia & 35 & 11.0 & 70 & 11.7 & 0.75 & 105 & 11.5 \\
\hline Do not know & 93 & 29.2 & 345 & 57.7 & $<0.0001$ & 438 & 47.8 \\
\hline \multicolumn{8}{|l|}{ After use, the woman can wash } \\
\hline 20 minutes after intercourse & 73 & 22.9 & 100 & 16.7 & 0.001 & 173 & 18.9 \\
\hline 2 hours after intercourse & 47 & 14.7 & 57 & 9.5 & & 104 & 11.3 \\
\hline 6 hours after intercourse & 20 & 6.3 & 33 & 5.5 & & 53 & 5.8 \\
\hline Do not know when & 158 & 49.5 & 387 & 64.7 & & 545 & 59.4 \\
\hline \multicolumn{8}{|l|}{ Side-effects? } \\
\hline Yes & 56 & 17.6 & 81 & 13.5 & $<0.0001$ & 137 & 14.9 \\
\hline No & 57 & 17.9 & 42 & 7.0 & & 99 & 10.8 \\
\hline Do not know & 190 & 59.6 & 454 & 75.9 & & 644 & 70.2 \\
\hline
\end{tabular}

Details about contraceptive type were filled in only by those who declared knowing about the contraceptive in question; percentages were calculated accordingly.

Some totals $<100 \%$ because of missing values. 


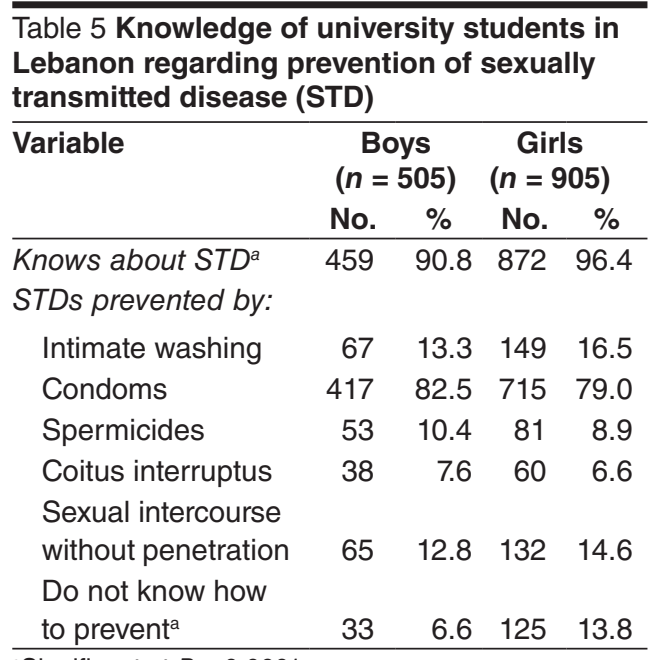

aSignificant at $P<0.0001$.

stinence should be practised for natural fertility regulation $(P<0.05$ for all). However, the majority of females declared they knew about the Ogino-Knauss (rhythm), Billings and temperature methods, although their knowledge lacked precision in most cases.

\section{Practices regarding sexual habits and contraception}

We found that $135(26.7 \%)$ males and 708 (78.2\%) females declared never having had a sexual relationship. Of those who had, around two-thirds of males and a quarter of females had used contraception, mainly the condom for males $(86.1 \%)$ and oral contraceptives for females $(56.3 \%)$ (Table 7$)$.

Sexual intercourse occurred most often at home in the absence of parents $(54.3 \%)$ or in a beach chalet $(49.6 \%)$ (Table 6$)$. In sexually active individuals, about $48 \%$ of males and $60 \%$ of females had ever had sexual relationships without using a contraceptive; the chief declared reason was extra-vaginal intercourse. Nevertheless, 34\% of all respondents thought that intercourse without penetration could lead to pregnancy.
The majority of those who had sexual experience would not seek help from health professionals for contraception; however, females who would stated they would mainly ask a gynaecologist and males a pharmacist. Half of all sexually active individuals would opt for an abortion if pregnancy occurred, and a quarter would use the morning after pill (Table 7).

\section{Discussion}

In this study, we found a low level of knowledge of contraception. We had expected a higher level of knowledge in this educated group of the Lebanese population. Greater knowledge of contraceptive issues was found among the better-educated women and men in Greece [7]. Our sample also revealed low use of contraceptive methods, particularly for females. This is in contrast to a study conducted in Brazil among 952 university undergraduates aged up to 24 years, where contraceptive use was high, especially for condoms and the pill [9]. Accordingly, in young Lebanese having a lower education, we would anticipate much lower levels of knowledge and use of contraception: this accounts for the urgency of the problem in Lebanese society.

Sources of sexual information were mainly friends, books, school and mass media, with $0 \%$ for health professionals. The mass media are known to shape attitudes and beliefs in young people [10], and it is becoming more accessible to Lebanese youth. In addition to printed media, there is high access to material on the Internet and television [10]. Furthermore, friends play a critical role in the sexual behaviours of adolescents [11]. This source of information is expected to be of low quality [7].

In our study, the majority of males and a very few females declared having had sex- 


\begin{tabular}{|c|c|c|c|c|c|c|c|}
\hline \multirow[t]{2}{*}{ Question } & \multicolumn{2}{|c|}{ Males $(n=505)$} & \multicolumn{2}{|c|}{ Females $(n=905)$} & \multirow[t]{2}{*}{ P-value } & \multicolumn{2}{|c|}{ Total $(n=1410)$} \\
\hline & No. & $\%$ & No. & $\%$ & & No. & $\%$ \\
\hline \multicolumn{8}{|l|}{ Ogino-Knauss method } \\
\hline Declares knowing about it & 333 & 65.9 & 732 & 80.9 & $<0.0001$ & 1065 & 75.5 \\
\hline $\begin{array}{l}\text { Menstrual cycle is from: } \\
1 \text { st day of menses to } 1 \mathrm{st}\end{array}$ & & & & & & & \\
\hline day of next menses & 117 & 35.1 & 407 & 55.6 & $<0.0001$ & 524 & 49.2 \\
\hline $\begin{array}{l}\text { Last day of menses to } 1 \mathrm{st} \\
\text { day of next menses }\end{array}$ & 66 & 19.8 & 153 & 20.9 & & 219 & 20.6 \\
\hline $\begin{array}{l}\text { 1st day of menses to last } \\
\text { day of next menses }\end{array}$ & 40 & 12.0 & 39 & 5.3 & & 79 & 7.4 \\
\hline Do not know & 91 & 27.3 & 101 & 13.8 & & 192 & 18.0 \\
\hline \multicolumn{8}{|l|}{ Ovulation occurs:: } \\
\hline 14 days after menses & 113 & 33.9 & 322 & 44.0 & 0.003 & 435 & 40.8 \\
\hline 14 days before menses & 76 & 22.8 & 228 & 31.1 & 0.007 & 304 & 28.5 \\
\hline $\begin{array}{l}\text { In general, at the mid } \\
\text { cycle }\end{array}$ & 89 & 26.7 & 176 & 24.0 & 0.31 & 265 & 24.9 \\
\hline It varies with women & 27 & 8.1 & 48 & 6.6 & 0.34 & 75 & 7.0 \\
\hline During menstruation & 14 & 4.3 & 15 & 2.0 & 0.04 & 29 & 2.7 \\
\hline Do not know & 64 & 19.2 & 62 & 8.5 & $<0.0001$ & 126 & 11.8 \\
\hline \multicolumn{8}{|l|}{$\begin{array}{l}\text { Abstinence should be } \\
\text { practised: }\end{array}$} \\
\hline On the day of ovulation & 56 & 16.8 & 144 & 19.7 & 0.24 & 200 & 18.8 \\
\hline One week before menses & 37 & 11.1 & 61 & 8.3 & 0.16 & 98 & 9.2 \\
\hline One week after menses & 46 & 13.8 & 51 & 7.0 & $<0.0001$ & 97 & 9.1 \\
\hline 1 week around ovulation & 71 & 21.3 & 313 & 42.8 & $<0.0001$ & 384 & 36.1 \\
\hline Do not know & 127 & 38.1 & 207 & 28.3 & 0.002 & 334 & 31.4 \\
\hline \multicolumn{8}{|l|}{ Billings method } \\
\hline $\begin{array}{l}\text { Declares knowing about it } \\
\text { Cervical secretions are: }\end{array}$ & 251 & 49.7 & 611 & 67.5 & $<0.0001$ & 862 & 61.1 \\
\hline $\begin{array}{l}\text { Abundant during ovulation } \\
\text { Abundant away from }\end{array}$ & 66 & 26.3 & 314 & 51.4 & $<0.0001$ & 380 & 44.1 \\
\hline ovulation & 30 & 12.0 & 67 & 11.0 & 0.67 & 97 & 11.3 \\
\hline Transparent during & & & & & & & \\
\hline ovulation & 56 & 22.3 & 163 & 26.7 & 0.19 & 219 & 25.4 \\
\hline $\begin{array}{l}\text { Transparent away from } \\
\text { ovulation }\end{array}$ & 34 & 13.5 & 87 & 14.2 & 0.80 & 121 & 14.0 \\
\hline $\begin{array}{l}\text { Elastic during ovulation } \\
\text { Elastic away from }\end{array}$ & 40 & 15.9 & 207 & 33.9 & $<0.0001$ & 247 & 28.7 \\
\hline ovulation & 14 & 5.6 & 50 & 8.2 & 0.19 & 64 & 7.4 \\
\hline Do not know & 96 & 38.2 & 149 & 24.4 & $<0.0001$ & 245 & $\underline{28.4}$ \\
\hline
\end{tabular}


Table 6 Knowledge regarding menstrual cycle and regulation of natural fertility (concluded)

\begin{tabular}{lccccccc}
\hline Question & \multicolumn{2}{c}{ Males $(\boldsymbol{n}=\mathbf{5 0 5})$} & \multicolumn{2}{c}{ Females $(\boldsymbol{n}=\mathbf{9 0 5})$} & $\boldsymbol{P}$-value & \multicolumn{2}{c}{ Total $(\boldsymbol{n}=\mathbf{1 4 1 0})$} \\
& No. & $\%$ & No. & $\%$ & & No. & $\%$ \\
\hline $\begin{array}{l}\text { Temperature method } \\
\text { Declares knowing about it }\end{array}$ & 285 & 56.4 & 656 & 72.5 & $<0.0001$ & 941 & 66.7 \\
$\begin{array}{l}\text { Temperature taken: } \\
\quad \text { Before getting up in }\end{array}$ & & & & & & & \\
$\quad$ morning & 139 & 48.8 & 448 & 68.3 & $<0.0001$ & 587 & 62.4 \\
$\quad$ Any time of the day & 55 & 19.3 & 65 & 9.9 & $<0.0001$ & 120 & 12.8 \\
$\quad$ Always by same route & 72 & 25.3 & 239 & 36.4 & 0.001 & 311 & 33.0 \\
$\quad$ With same thermometer & 74 & 26.0 & 222 & 33.8 & 0.02 & 296 & 31.5 \\
$\quad$ Is higher after ovulation & 106 & 37.2 & 309 & 47.1 & 0.006 & 415 & 44.1 \\
$\quad$ Is lower after ovulation & 30 & 10.5 & 101 & 15.4 & 0.05 & 131 & 13.9 \\
Coitus interruptus & & & & & & & \\
Declares knowing about it & 354 & 70.1 & 612 & 67.6 & 0.25 & 966 & 68.5 \\
Ejaculation away from vulva & 246 & 69.5 & 409 & 66.8 & 0.35 & 655 & 67.8 \\
\hline
\end{tabular}

Details about contraceptive type were filled in only by those who declared knowing about the contraceptive in question; percentages were calculated accordingly.

Some totals $<100 \%$ because of missing values.

ual relations. The reasons for the difference may include differences in socioeconomic status in the sample, since it is constituted of more females from public universities, and lower socioeconomic status is associated with higher religiosity in young Lebanese adults [12]. Differences between males and females in religiosity or inequity in sexual experiences in the view of society are also possible. Religiosity is known to affect sexual behaviour, with more frequent attendance at religious services and stronger religious beliefs associated with delaying sexual initiation $[5,13]$. Indeed, Lebanese females are particularly religious [12], and the Lebanese society encourages sexual experience for males but prohibits it for females.

With these reported low levels of knowledge, systematic and responsible education in the promotion of good reproductive health is very important in Lebanon. Policymakers should recognize the importance of designing interventions that give adolescents the skills they need to feel effective in their ability to communicate about sex and contraception [14]. Although family planning efforts have generally been conducted through public facilities, some countries are now placing a significant emphasis on private channels of delivery [10], focusing work on fostering youngsters' identities and promoting their ability to take care of themselves [3]. Findings in conservative societies have even shown the normative influence of parents, older family members and extended family members, even in sexual decision-making [2]. Accordingly, prevention approaches should concentrate on providing information and motivation for abstinence or safer sex [15].

Lebanese students have cited having sex mainly in the absence of their parents. However, there is an association between the amount of unsupervised time and sexual behaviours, with STD rates suggestive of particularly risky sexual behaviours [16] As youths come of age, parents probably believe that it is appropriate to leave them increasingly on their own. However, parents 


\begin{tabular}{|c|c|c|c|c|c|c|c|}
\hline \multirow[t]{2}{*}{ Question $^{\mathrm{a}}$} & \multicolumn{2}{|c|}{ Males $(n=505)$} & \multicolumn{2}{|c|}{ Females $(n=905)$} & \multirow[t]{2}{*}{$P$-value } & \multicolumn{2}{|c|}{ Total $(n=1410)$} \\
\hline & No. & $\%$ & No. & $\%$ & & No. & $\%$ \\
\hline \multicolumn{8}{|l|}{ Already had sexual relations } \\
\hline No, never & 135 & 26.7 & 708 & 78.2 & $<0.0001$ & 843 & 59.8 \\
\hline Yes, with vaginal penetration & 241 & 47.7 & 63 & 7.0 & $<0.0001$ & 304 & 21.6 \\
\hline Already used contraception ${ }^{b}$ & 237 & 64.1 & 48 & 24.4 & $<0.0001$ & 285 & 50.3 \\
\hline Yes, without penetration & 137 & 27.1 & 78 & 8.6 & 0.005 & 215 & 15.2 \\
\hline Male condom ${ }^{c}$ & 204 & 86.1 & 2 & 4.2 & $<0.0001$ & 206 & 72.3 \\
\hline Yes, with anal penetration & 128 & 25.3 & 28 & 3.1 & $<0.0001$ & 156 & 11.1 \\
\hline Oral contraceptive $^{c}$ & - & - & 27 & 56.3 & & 27 & 9.5 \\
\hline Spermicidal products ${ }^{c}$ & 2 & 0.8 & 2 & 4.2 & & 4 & 1.4 \\
\hline Coitus interruptus ${ }^{c}$ & 1 & 0.4 & 1 & 2.1 & & 2 & 0.7 \\
\hline \multicolumn{8}{|l|}{$\begin{array}{l}\text { Would seek help for } \\
\text { contraception }^{b}\end{array}$} \\
\hline No & 231 & 62.4 & 119 & 60.4 & 0.02 & 250 & 44.1 \\
\hline Yes, gynaecologist & 24 & 6.5 & 63 & 32.0 & $<0.0001$ & 87 & 15.3 \\
\hline Yes, pharmacist & 47 & 12.7 & 13 & 6.6 & $<0.0001$ & 60 & 10.6 \\
\hline Yes, general physician & 31 & 8.4 & 10 & 5.1 & 0.003 & 41 & 7.2 \\
\hline Yes, midwife & 11 & 3.0 & 12 & 6.1 & 0.46 & 23 & 4.1 \\
\hline Yes, social worker & 12 & 3.2 & 6 & 3.0 & 0.30 & 18 & 3.2 \\
\hline Yes, nurse & 7 & 1.9 & 8 & 4.1 & 0.50 & 15 & 2.6 \\
\hline \multicolumn{8}{|l|}{ Had sex without contraception ${ }^{b}$} \\
\hline No & 193 & 52.2 & 80 & 40.6 & 0.004 & 273 & 48.1 \\
\hline Yes, extra-vaginal & 60 & 16.2 & 43 & 21.8 & 0.55 & 103 & 18.2 \\
\hline Yes, because unplanned & 45 & 12.2 & 25 & 12.7 & 0.40 & 70 & 12.3 \\
\hline Yes, during infertile days & 35 & 9.5 & 30 & 15.2 & 0.20 & 65 & 11.5 \\
\hline \multicolumn{8}{|l|}{ Where do you usually have sex } \\
\hline Home, parents absent & 203 & 54.9 & 105 & 53.3 & 0.30 & 308 & 54.3 \\
\hline Beach chalet & 196 & 53.0 & 85 & 43.1 & 0.23 & 281 & 49.6 \\
\hline Car & 158 & 42.7 & 77 & 39.1 & 0.90 & 235 & 41.4 \\
\hline Dormitory rooms & 111 & 30.0 & 32 & 16.2 & 0.002 & 143 & 25.2 \\
\hline Beach & 103 & 27.8 & 36 & 18.3 & 0.05 & 139 & 24.5 \\
\hline Forest & 94 & 25.4 & 32 & 16.2 & 0.05 & 126 & 22.2 \\
\hline Night club & 82 & 22.2 & 30 & 15.2 & 0.15 & 112 & 19.8 \\
\hline Home, parents present & 50 & 13.5 & 33 & 16.8 & 0.12 & 83 & 14.6 \\
\hline Movie theatre & 58 & 15.7 & 24 & 12.2 & 0.50 & 82 & 14.5 \\
\hline University & 44 & 11.9 & 17 & 8.6 & 0.41 & 61 & 10.8 \\
\hline Public place (road, garden) & 30 & 8.1 & 18 & 9.1 & 0.42 & 48 & 8.5 \\
\hline \multicolumn{8}{|l|}{ Thinks sex without penetration } \\
\hline can lead to pregnancy & 173 & 34.3 & 310 & 34.3 & 0.94 & 483 & 34.3 \\
\hline Would abort a pregnancy ${ }^{b}$ & 187 & 50.5 & 102 & 51.8 & 0.06 & 289 & 51.0 \\
\hline Would use morning after pill ${ }^{b}$ & 84 & 22.7 & 49 & 24.9 & 0.86 & 133 & 23.5 \\
\hline
\end{tabular}

Some totals $<100 \%$ because of missing values.

${ }^{a}$ More than one answer possible.

${ }^{b}$ Percentages calculated for those who declared previous sexual relations.

cPercentages calculated for those who used contraception. 
and community members should consider increasing opportunities for supervised activities, which could reduce risk-taking among youth [16].

We are aware of the possible biases that could arise from the study methodology: selection bias is possible due to the nature of the sample; however, we have no reason to believe that it would affect our results, since availability of students in the campus is theoretically unrelated to their sexual knowledge and practices. Refusal rate was acceptable $(30 \%)$, but we had no means of evaluating reasons for refusal. Information bias is also possible, as in all surveys; the sensitivity of the subject increases the risk of false answers, particularly regarding practices. However, we doubt that any measurement error would be sufficient to change the shape of our results. We believe that this study is reasonably able to depict the reality of contraception in young Lebanese university students, but the results cannot be extrapolated to other young Lebanese.

\section{References}

1. Erulkar A et al. Behavior change evaluation of a culturally consistent reproductive health program for young Kenyans. International family planning perspectives, 2004, 30(2):58-67.

2. Dei $\mathrm{M}$ et al. The resistance to contraceptive use in young Italian women. European journal of contraception and reproductive health care, 2004, 9:214-20.

3. Villarruel A et al. Predictors of sexual intercourse and condom use intentions among Spanish-dominant Latino youth: a test of the planned behavior theory. Nursing research, 2004, 53(3):172-81.

4. Gokengin D et al. Sexual knowledge, attitudes and risk behaviors of students in Turkey. Journal of school health, 2003, 73(7):258-63.

5. Manlove J, Ryan S, Franzetta K. Contraceptive use and consistency in U.S. teenagers' most recent sexual relationships. Perspectives on sexual and reproductive health, 2004, 36(6):265-75.

6. Bender S, Kosunen E. Teenage contraceptive use in Iceland: a gender perspective. Public health nursing, 2005, 22(1):17-26.

7. Tountas $Y$ et al. Information sources and level of knowledge of contraception issues among Greek women and men in the reproductive age: a country-wide survey. European journal of contraception and reproductive health care, 2004, 9:1-10.

8. Glover EK et al. Sexual health experiences of adolescents in three Ghanaian towns. International family planning perspectives, 2003, 29(1):32-40.

9. Machado Pirotta KC, Schor N. Intenções reprodutivas e práticas de regulação da fecundidade entre universitários [Reproductive intentions and fertility regulation practices among university students]. $R e$ vista de saúde pública, 2004, 38(4):495502.

10. Katende C, Gupta N, Bessinger R. Facility-level reproductive health interventions and contraceptive use in Uganda. International family planning perspectives, 2003, 29(3):130-7.

11. Harper $\mathrm{G}$ et al. The role of close friends in African American adolescents' dating and sexual behavior. Journal of sex research, 2004, 41(4):351-62.

12. Baalbaky G. Religiosity in Lebanese youth. In: God and the right to the difference. Kaslik, Lebanon, Holy Spirit University, 2005. 
13. Rostosky SS et al. Coital debut: the role of religiosity and sex attitudes in the Add Health Survey. Journal of sex research, 2003, 40(4):358-67.

14. Halpem-Felsher B et al. Adolescents' self efficacy to communicate about sex: its role in condom attitudes, commitment and use. Adolescence, 2004, 39(155):44356.
15. Eisenberg $M$ et al. Parents' beliefs about condoms and oral contraceptives: are they medically accurate? Perspectives on sexual and reproductive health, 2004, 36(2):50-7.

16. Cohen $\mathrm{D}$ et al. When and where do youths have sex? The potential role of adult supervision. Pediatrics, 2002, 110(6):6674.

\section{World Health Day 2009: Save lives. Make hospitals safe in emer- gencies}

World Health Day 2009 focuses on the safety of health facilities and the readiness of health workers who treat those affected by emergencies. Health centres and staff are critical life-lines for vulnerable people in disasters - treating injuries, preventing illnesses and caring for people's health needs.

They are cornerstones for primary health care in communities-meeting everyday needs, such as safe childbirth services, immunizations and chronic disease care that must continue in emergencies. Often, already fragile health systems are unable to keep functioning through a disaster, with immediate and future public health consequences.

World Health Day is one of WHO's most visible opportunities to raise awareness of global health priorities. This year, WHO and international partners will underscore the importance of investing in health infrastructure that can withstand hazards and serve people in immediate need. They will also urge health facilities to implement systems to respond to internal emergencies, such as fires, and ensure the continuity of care. 